\title{
SCINTILLATION MONOCRYSTALS OF KDP:TI AND KDP:Ce DOPED BY THALLIUM AND CERIUM FOR SELECTIVE DETECTION OF FAST NEUTRONS
}

\author{
A.P. Voronov ${ }^{1}$, S.V. Naydenov ${ }^{1}$, I.M. Pritula ${ }^{1}$, \\ G.M. Onyshchenko, ${ }^{2 * 3}$, A.F. Shchus' ${ }^{2}$, I.I. Yakymenko ${ }^{2}$ \\ ${ }^{1}$ Institute for Single Crystals, National Academy of Sciences of Ukraine \\ 60 Nauky Ave., Kharkiv, 61001, Ukraine \\ ${ }^{2}$ V.N. Karazin Kharkiv National University \\ 6 Svobody Sq., Kharkiv, 61022, Ukraine \\ ${ }^{3}$ Institute for Scintillation Materials, National Academy of Sciences of Ukraine \\ 60 Nauky Ave., Kharkiv, 61001, Ukraine \\ *E-mail: gennadiy.m.onyshchenko@karazin.ua, https://orcid.org/0000-0001-6945-8413 \\ Received 9 June 2018; revised 15 July 2018; accepted 10 September 2018
}

This research is going to explain the fast neutrons and gamma radiation registration by the new inorganic single crystals of the KDP (Potassium Dihydrogen Phosphat) group that was grown from the water solutions and activated by the thallium $\mathrm{Tl}^{+}$or cerium $\mathrm{Ce}^{3+}$. The appearance of the luminescence upon KDP:Tl crystals irradiation with the fast neutrons is explained by secondary ionizing radiation (recoil protons and oxygen recoil nuclei) with excitation of the activator under ionization losses for inhibition. Also the recombination mechanism with the radiation defects of the hydrogen sublattice going to transmit electron excitations to the region of the $\mathrm{Tl}^{+}$luminescence center. In the KDP:Ce crystals the excitation mechanism of the activator has a similar character, but the luminescence itself is due to the $5 \mathrm{~d} \rightarrow 4 \mathrm{f}$ transition in $\mathrm{Ce}^{3+}$ ions. The detection efficiency for activated KDP:Tl and KDP:Ce crystals to the fast neutrons in comparisons with organic (plastic) scintillators were calculated theoretically and experimentally. For the crystal volume about 10x10x10 mm3 with the optimal activator concentration the fast neutron detection efficiency under irradiation of ${ }^{239} \mathrm{Pu}-\mathrm{Be}$ is $12 \%$ for KDP:Tl and $16 \%$ for KDP:Ce, which is in a consistent with the theoretical calculation and is not lower according to the parameters of typical organic scintillators. A high natural selectivity of the KDP scintillators to the fast neutrons due to their low sensitivity to gamma radiation was detected. Herewith the internal discrimination of $\mathrm{n} / \gamma$ signals (the ratio of detection efficiencies for the fast neutrons and gamma quants) for activated KDP crystals is 7-8 times higher than of regular plastic scintillators.

KEYWORDS: fast neutrons, KDP-type single crystals, scintillation detectors, detection efficiency, neutron/gamma discrimination

\author{
СЦИНТИЛЯЦІЙНІ МОНОКРИСТАЛИ КDР:TІ I КDР:Се \\ АКТИВОВАНІ ТАЛІЕМ І ЦЕРІЕМ ДЛЯ СЕЛЕКТИВНОГО ДЕТЕКТУВАННЯ \\ ШВИДКИХ НЕЙТРОНІВ \\ О.П. Воронов ${ }^{1}$, С.В. Найдьонов ${ }^{1}$, І.М. Притула ${ }^{1}$, \\ Г.М. Онищенко ${ }^{2,3}$, О.П. Щусь ${ }^{2}$, І.І. Якименко ${ }^{2}$ \\ ${ }^{1}$ Інститут монокристалів, Національна академія наук Украӥни \\ 61001, Україна, м. Харків, пр. Науки, 60 \\ ${ }^{2}$ Харківський національний університет імені В.Н. Каразіна \\ 61022, Україна, м. Харків, м. Свободи, 4 \\ ${ }^{3}$ Інститут сцинтиляційних матеріалів, Національна академія наук Украйни \\ 61001, Україна, м. Харків, пр. Науки, 60
}

Досліджено детектування швидких нейтронів і гамма-випромінювання новими неорганічними монокристалами групи KDP, активованих талієм $\mathrm{Tl}^{+}$або церієм $\mathrm{Ce}^{3+}$ i вирощених 3 водних розчинів. Виникнення люмінесценції при опроміненні швидкими нейтронами в сцинтиляційних кристалах KDP:Tl обумовлено вторинним іонізуючим випромінюванням (протони віддачі і ядра віддачі кисню) із збудженням активатора при іонізаційних втратах на гальмування, а також рекомбінаційним механізмом за участю радіаційних дефектів водневої підгратки, що передає електронні збудження в околицю центру світіння $\mathrm{Tl}^{+}$. У кристалах $\mathrm{KDP}: \mathrm{Ce}$ механізм збудження активатора має аналогічний характер, але сама люмінесценція зумовлена переходом $5 \mathrm{~d} \rightarrow$ 4f в іонах $\mathrm{Ce}^{3+}$. Для активованих кристалів KDP:Tl i KDP:Се теоретично і експериментально визначено ефективність детектування швидких нейтронів, а також проведено порівняння з органічними (пластиковими) сцинтиляторами. Для оброблених кристалів розміром 10х10х10 мм 3 оптимальною концентрацією активатора ефективність детектування швидких нейтронів при опроміненні ${ }^{239} \mathrm{Pu}-\mathrm{Be}$ джерелом досягає $12 \%$ для KDP:Tl і 16\% для KDP:Cе, що добре узгоджується з наведеними теоретичними розрахунками і $є$ близькою до параметрів поширених органічних сцинтиляторів. Виявлена висока природна селективність KDP-сцинтиляторів при детектуванні швидких нейтронів за рахунок їх низької чутливості до гамма-випромінювання. При цьому внутрішній поділ $\mathrm{n} / \gamma$ сигналів (відношення ефективностей детектування при реєстрації швидких нейтронів і гамма-квантів) для активованих кристалів KDP в 7-8 разів перевищує параметри традиційних пластикових сцинтиляторів.

КЛЮЧОВІ СЛОВА: швидкі нейтрони, КDР-монокристали, сцинтиляційні детектори, ефективність детектування, нейтрон/гамма дискримінація 


\section{СЦИНТИЛЛЯЦИОННЫЕ МОНОКРИСТАЛЛЫ КDР:ТІ И КDР:СР АКТИВИРОВАННЫЕ ТАЛЛИЕМ И ЦЕРИЕМ ДЛЯ СЕЛЕКТИВНОГО ДЕТЕКТИРОВАНИЯ БЫСТРЫХ НЕЙТРОНОВ}

А.П. Воронов ${ }^{1}$, С.В. Найденов ${ }^{1}$, И.М. Притула ${ }^{1}$, Г.М. Онищенко ${ }^{2,3}$, А.Ф. Щусь ${ }^{2}$ И.И. Якименко ${ }^{2}$

${ }^{1}$ Институт монокристаллов, Национальная академия наук Украины 61001, Украина, г. Харьков, пр. Науки, 60

${ }^{2}$ Харьковский национальный университет имени В.Н. Каразина 61022, Украина, г. Харьков, пл. Свободы, 4

${ }^{3}$ Институт сцинтилляционных материалов, Национальная академия наук Украины 61001, Украина, г. Харьков, пр. Науки, 60

Исследовано детектирование быстрых нейтронов и гамма-излучения новыми неорганическими монокристаллами группы $\mathrm{KDP}$, активированных таллием $\mathrm{Tl}^{+}$или церием $\mathrm{Ce}^{3+}$ и выращенных из водных растворов. Возникновение люминесценции при облучении быстрыми нейтронами в сцинтилляционных кристаллах KDP:Tl обусловлено вторичным ионизирующим излучением (протоны отдачи и ядра отдачи кислорода) с возбуждением активатора при ионизационных потерях на торможение, а также рекомбинационным механизмом с участием радиационных дефектов водородной подрешетки, передающих электронные возбуждения в окрестность центра свечения $\mathrm{Tl}^{+}$. В кристаллах KDP:Се механизм возбуждения активатора имеет аналогичный характер, но сама люминесценция обусловлена переходом $5 \mathrm{~d} \rightarrow 4 \mathrm{f}$ в ионах Се ${ }^{3+}$. Для активированных кристаллов KDP:Tl и KDP:Cе теоретически и экспериментально определена эффективность детектирования быстрых нейтронов, а также проведено сравнение с органическими (пластмассовыми) сцинтилляторами. Для обработанных кристаллов размером $10 \times 10 \times 10$ мм $^{3}$ с оптимальной концентрацией активатора эффективность детектирования быстрых нейтронов при облучении ${ }^{239} \mathrm{Pu}-\mathrm{Be}$ источником достигает $12 \%$ для KDP:T1 и $16 \%$ для KDP:Cе, что находится в хорошем согласии с приведенным теоретическим расчетом и не уступает параметрам распространенных органических сцинтилляторов. Обнаружена высокая естественная селективность КDP-сцинтилляторов при детектировании быстрых нейтронов за счет их низкой чувствительности к гамма-излучению. При этом внутреннее разделение $\mathrm{n} / \gamma$ сигналов (отношение эффективностей детектирования при регистрации быстрых нейтронов и гамма-квантов) для активированных кристаллов KDP в 7-8 раз превосходит параметры традиционных пластиковых сцинтилляторов.

КЛЮЧЕВЫЕ СЛОВА: быстрые нейтроны, КDР-монокристаллы, сцинтилляционные детекторы, эффективность детектирования, нейтрон/гамма дискриминация

Несмотря на разнообразие типов разработанных к настоящему времени детекторов быстрых нейтронов [1-5] и используемых в них твердых, жидких и газообразных детектирующих сред, задача эффективной раздельной регистрации быстрых нейтронов в смешанных $\mathrm{n} / \gamma$ радиационных полях, создаваемых различными ядерными источниками, в полной мере не решена и остается весьма актуальной. Как правило, многие из материалов, которые хорошо регистрируют быстрые нейтроны, в том числе по реакции упругого рассеяния с образованием протонов отдачи [6], одновременно обладают излишней чувствительностью к сопутствующему (или фоновому) гамма-излучению [7,8]. Из-за этого для раздельной регистрации нейтронов приходится дополнительно использовать непростые методы разделения аппаратных сигналов по форме, амплитуде и длительности импульсов. Поэтому поиск новых детектирующих материалов, которые изначально обладают высокой внутренней селективностью к нейтронам и гамма-излучению, представляет особый интерес для многих радиационных приложений.

К числу методов регистрации быстрых нейтронов относится метод детектирования протонов отдачи, возникающих при упругом рассеянии нейтронов в водородсодержащих средах. Для многих приложений достаточно ограничиться применением быстрых нейтронов с энергиями от $100 \mathrm{keV}$ до $10 \mathrm{MeV}$. Их детектирование можно осуществить в твердых или жидких водородосодержащих сцинтилляторах. Наибольшее распространение получили твердотельные детекторы с использованием органических (молекулярных) кристаллов антрацена, стильбена и др. [9] или пластмассовых сцинтилляторов на основе полистирола, полиметилметакрилата и др. с активаторами и спектросмещающими добавками. Однако все эти детекторы имеют высокую чувствительность к сопутствующему гамма-излучению низких и средних энергий. Для того, чтобы исключить его влияние, в таких детекторах используют различные методы пассивной и активной защиты, а также метод разделения сигналов по форме импульса [10].

Недавно в Институте монокристаллов (Харьков, Украина) удалось вырастить из водных растворов сцинтилляционные кристаллы дигидрофосфата калия $\mathrm{KH}_{2} \mathrm{PO}_{4}(\mathrm{KDP})$, допированные ионами таллия $\mathrm{Tl}^{+}$или церия $\mathrm{Ce}^{3+}$ [11-14]. Уникальность полученных кристаллов KDP:T1 и KDP:Се состоит в том, что они занимают промежуточное положение между органическими и неорганическими сцинтилляторами. С одной стороны, каждый кристалл из семейства $\mathrm{KDP}$ - это типичный широкозонный $\left(E_{g}=8,8\right.$ эВ) неорганический диэлектрик с сильными ковалентными связями в фосфатной анионной группе $\mathrm{H}_{2} \mathrm{PO}_{4}^{-}$, связанной менее сильной ионной связью с катионной подрешеткой ионов $\mathrm{K}^{+}$. С другой стороны, этот кристалл содержит высокоподвижную (при комнатных температурах) подрешетку атомов водорода, насыщенную многочисленными водородными связями с близлежащими атомами кислорода. Концентрация ядер водорода в решетке KDP составляет $2,07 \times 10^{22} \mathrm{~cm}^{-3}$. Это примерно вдвое меньше, чем концентрация атомов водорода в пластиковых (полистирол и др.) или молекулярных (антрацен и др.) органических сцинтилляторах. Однако, как следует из приведенных далее расчетов и экспериментов, этого вполне достаточно для эффективной регистрации быстрых нейтронов. 
Основная цель данной работы - исследовать сцинтилляционные свойства и возможное применение активированных таллием и церием кристаллов KDP:Tl и KDP:Cе в качестве эффективных сред для селективного детектирования быстрых нейтронов в смешанных n/ $\gamma$ радиационных полях.

\section{ВЫРАЩИВАНИЕ СЦИНТИЛЛЯЦИОННЫХ МОНОКРИСТАЛЛОВ КDР:ТL И КDР:СЕ}

Чистые (не активированные различными добавками) неорганические кристаллы KDP традиционно выращивают из водных растворов методом снижения температуры. Таким методом могут быть выращены кристаллы KDP очень больших размеров с апертурой вплоть до 50х50 см² и весом до 500 кг [15]. Чистые кристаллы имеют широкую полосу оптической прозрачности (175-1500 нм), высокое структурное совершенство (плотность дислокаций $<10^{2} \mathrm{~cm}^{-2}$ ) и высокую радиационную прочность к действию различных видов ионизирующего излучения. Коэффициент оптического поглощения в этих кристаллах не изменяется при облучении мощными потоками протонов с флюенсом до $10^{13}$ протон/см², быстрых нейтронов до $10^{10}$ нейтрон/см² или гамма-частиц - с дозовой нагрузкой вплоть до $10^{5} \mathrm{~Gy}\left(10^{7}\right.$ рад) [16]. Отметим, что именно наличие в структуре KDP обширных водородных связей позволяет достаточно легко включать в матрицу кристалла различные неорганические и органические добавки [17].

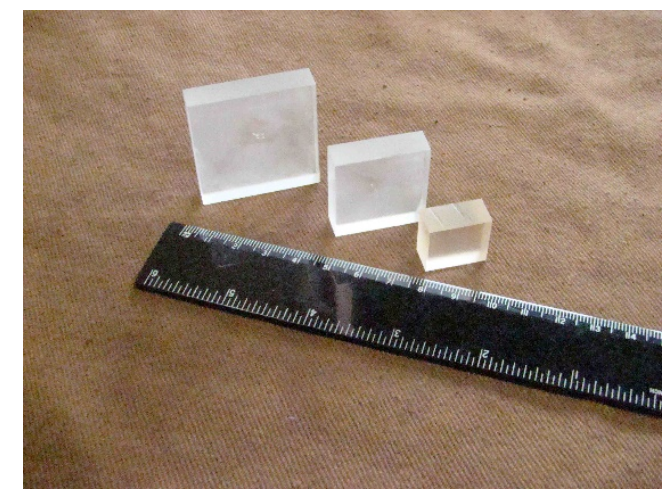

Рис. 1. Образцы обработанных кристаллов KDP:T1 с различной концентрацией активатора (слева и посередине) и KDP:Се (справа) разного размера.

Монокристаллы KDP:Tl и KDP:Се выращивали из водного раствора (с пересыщением не менее 5\%) методом снижения температуры в интервале $40-60^{\circ} \mathrm{C}$. Соль $\mathrm{KH}_{2} \mathrm{PO}_{4}$ синтезировали из реагентов $\mathrm{KOH}_{\text {и }} \mathrm{H}_{3} \mathrm{PO}_{4}$ квалификации «extra pure substance». Допант $\mathrm{Tl}^{+}$в концентрации $10^{-2}, 10^{-1}$ и 1,0 wt. \% вводили в маточный раствор в виде водного раствора соли $\mathrm{TlNO}_{3}$. Прямое допирование церием в виде водного раствора $\mathrm{Ce}\left(\mathrm{NO}_{3}\right)_{3}$ было невозможно из-за образования нерастворимого в воде фосфата церия $\mathrm{CePO}_{4}$. Поэтому допант Се ${ }^{3+}$ в концентрации $10^{-2}$ wt.\% вводили [14] в маточный раствор в виде водного раствора органо-металлического комплекса (АC-Ce) вида $\left(\mathrm{C}_{19} \mathrm{H}_{15} \mathrm{NO}_{8}-\mathrm{Ce}^{3+}\right)$, где $\mathrm{C}_{19} \mathrm{H}_{15} \mathrm{NO}_{8}-$ органический лиганд ализарин-комплексон (АС). Содержание таллия в секторах призмы и пирамиды оказалось примерно одинаковым. Следовательно, примесь распределяется по секторам роста относительно равномерно. Содержание церия в секторах роста призмы и пирамиды оказалось разным - равным $10^{-2}$ wt.\% и $5 \times 10^{-3}$ wt. \%, соответственно.

На рис. 1 показаны вырезанные из заготовок и обработанные монокристаллы KDP:Tl и KDP:Ce типичных размеров, которые были использованы в последующих измерениях.

\section{МЕХАНИЗМ СЦИНТИЛЛЯЦИОННОГО ОТКЛИКА В АКТИВИРОВАННЫХ КРИСТАЛЛАХ КDР}

Рассмотрим природу свечения и механизм люминесценции в кристаллах $\mathrm{KDP}: \mathrm{Tl}^{+}$. Ион таллия $\mathrm{Tl}^{+}-э$ хорошо известный активатор, формирующий электронные $\mathrm{s}^{2}$-центры в кубических щелочно-галоидных кристаллах [18]. Центр $\mathrm{Tl}^{+}$в кристаллах $\mathrm{KDP}: \mathrm{Tl}^{+}-$это единственный подтвержденный $s^{2}$-центр в некубической собственной решетке, для которого возможны $s^{2} \rightarrow s p$ переходы, соответствующие полосам поглощения в обычных щелочно-галоидных кристаллах [19].

Возбуждение люминесценции под воздействием рентгеновских лучей в кристаллах KDP:Tl было рассмотрено в работе [20], в которой предложена модель рекомбинационного механизма люминесценции. Ионизирующее излучение, как обычно, приводит к генерации основных носителей заряда - зонных электронов и дырок и образованию связанных электронно-дырочных пар. Кроме того, под его воздействием в водородной подрешетке кристаллов KDP:Tl возникают пары противоположных френкелевских дефектов в виде межузельного иона $\mathrm{H}^{+}$(D-дефект) и вакансии водорода $\mathrm{V}_{\mathrm{H}}^{+}$(отрицательно заряженный L-дефект). D-дефект после захвата зонного электрона превращается в межузельный нейтральный атом водорода - $\mathrm{H}^{0}$-центр, который обладает высокой подвижностью в кристалле. L-дефект, локализованный на анионе $\left(\mathrm{H}_{2} \mathrm{PO}_{4}\right)^{-}$, после захвата дырки превращается в другой центр захвата - А-радикал. Оба указанных центра в дальнейшем могут испытывать диффузию по решетке и рекомбинацию с основными зонными носителями заряда (Н ${ }^{0}$-центр 
с дыркой, а А-радикал с электроном, соответственно). В результате рекомбинации электрона и дырки заряженный центр возвращается в свое исходное состояние. При обычных условиях в широком температурном интервале эта рекомбинация является безизлучательной [21,22]. Но, перенос энергии, высвобождаемой во время такой рекомбинации, может приводить к возбуждению иона $\mathrm{Tl}^{+}$в случае, если центр захвата расположен в непосредственной близости от него. Кроме того, возбуждение иона $\mathrm{Tl}^{+}$может происходить непосредственно вторичными ионизирующими частицами, хотя доля переданной при этом энергии излучения будет не столь значительной, так как концентрация допанта является достаточно низкой. В конечном итоге во всех случаях спектральный состав радиолюминесценции кристалла $\mathrm{KDP}_{\mathrm{T}} \mathrm{Tl}^{+}$определяется низшим излучательным переходом таллия ${ }^{3} \mathrm{P}_{1} \rightarrow{ }^{1} \mathrm{~S}_{0}$ на основной уровень. Максимум радиолюминесценции соответствует 275 нм (4,5 эВ). Каскадные переходы с более высоких возбужденных уровней таллия на самый низкий возбужденный уровень остаются безизлучательными.

Сцинтилляционный отклик кристалла KDP:Се обусловлен аналогичным механизмом люминесценции. Отличия касаются переноса возбуждения на центр свечения, которым является ион церия Се $\mathrm{C}^{3+}$, и снятие с него возбуждения с высвечиванием импульса радиолюминесценции. Импульс радиолюминесценции кристаллов $\mathrm{KDP}: \mathrm{Ce}$ обусловлен конфигурационными переходами с нижнего уровня 5d-оболочки ионов Се $\mathrm{e}^{3+}$ на уровни основного состояния 4f-оболочки. Максимум спектра сцинтилляций соответствует $\lambda_{e m}=350 \mathrm{HM}(3,54$ эВ). В отличие от кристаллов KDP:Tl он сдвинут в длинноволновую область, что несколько увеличивает чувствительность (и эффективность) детектирования быстрых нейтронов при использовании обычных бищелочных фотоумножителей.

Особенности описанного выше рекомбинационного механизма сохраняются при рассмотрении люминесценции KDP:T1 и KDP:Се под облучением быстрыми нейтронами. Однако эффективность образования и концентрация решеточных дефектов внутри водородной подрешетки будет на порядок выше, так кристалл облучается тяжелыми частицами. При упругом рассеянии быстрых нейтронов с характерной энергией выше 0,5 МэВ в матрице KDP возникают ионизационные потоки отдачи легких ядер - протонов (при рассеянии нейтронов на ядрах водорода) и ядер кислорода. Атомы кислорода в решетке KDP связаны многочисленными водородными связями с атомами водорода. Поэтому отдача от ядер кислорода будет приводить к сравнимому эффекту ионизации и возбуждения водородной подрешетки, который производят одни протоны отдачи. Вкладом в передачу энергии ионизирующего излучения при отдаче более тяжелых ядер калия (атомный вес $A=39)$ и фосфора $(A=31)$ можно пренебречь, так как суммарные потери энергии нейтрона $E_{n}$ на этих ядрах пропорциональны $\Delta E_{n} \sim E_{n} / A$ и составляют менее $6 \%$ по сравнению с потерями на протонах отдачи. Кроме того, удельная тормозная способность и плотность ионизационных потерь при торможении ядер отдачи калия (заряд $Z=19)$ и фосфора $(Z=15)$ быстро растут с ростом заряда, что приводит к более значительному радиационному тушению люминесценции [7], чем при ионизационных потерях от выбитых ядер водорода и кислорода. При торможении протонов отдачи или легких ядер в веществе (с образованием вторичных быстрых $\delta$-электронов) в конечном итоге будет происходить ионизация и возбуждение атомов среды аналогично рентгеновскому облучению. Потолок валентной зоны кристалла KDP в основном заполнен состояниями 2p-орбиталей атомов кислорода, а потолок зоны проводимости - 1s-состояниями водорода. Ионизация указанных атомов будет приводить к генерации зонных носителей в кристалле. Прямая отдача первичных нейтронов и торможение вновь образованных вторичных заряженных частиц будут генерировать дефекты водородной подрешетки KDP.

\section{ТЕОРЕТИЧЕСКАЯ ОЦЕНКА ЭФФЕКТИВНОСТИ ДЕТЕКТИРОВАНИЯ БЫСТРЫХ НЕЙТРОНОВ} КDР-СЦИНТИЛЛЯТОРАМИ

Ослабление потока нейтральных частиц, фотонов в веществе происходит по экспоненциальному закону. В реакции упругого рассеяния каждый нейтрон может многократно испытывать потерю энергии при столкновениях с ядрами среды. Однако при рассеянии на легких ядрах нейтрон быстро теряет свою энергию и ядра отдачи, возникающие после нескольких столкновений, получают от него гораздо меньшую энергию и уже не вносят существенного вклада в сцинтилляционный отклик по сравнению с вкладом от однократного рассеяния быстрых нейтронов. Если принять, что регистрируется каждое ядро отдачи, и ограничиться вкладом однократного рассеяния, то эффективность детектирования можно записать в виде

$$
\varepsilon_{n}^{(1)}=\frac{\Sigma_{e l}}{\sum_{t o t}}\left[1-\exp \left(-\Sigma_{e l} h\right)\right]
$$

где $\Sigma_{e l}-$ макроскопическое сечение упругого рассеяния нейтронов, $\Sigma_{t o t}=\Sigma_{e l}+\Sigma_{\text {non-el }}-$ полное макроскопическое сечение; $h$ - толщина материала в направлении потока нейтронов. Макроскопическое сечение для $k$-го канала (реакции) нейтронов $\Sigma_{k}=\sum_{i} n_{i} \sigma_{i, k}$ определяется концентрациями $n_{i}$ ядер $i$-го сорта, входящих в состав среды и микроскопическими сечениями рассеяния $\sigma_{i, k}$, причем $\sigma_{i, \text { tot }}=\sigma_{i, e l}+\sigma_{i, n o n-e l}$. 
Для органических сцинтилляторов (пластик или органические монокристаллы) следует учесть, что перед рассеянием на ядрах водорода нейтрон может многократно рассеиваться на ядрах углерода, что вносит дополнительный вклад в полную эффективность детектирования. В результате суммирования вкладов многократного рассеяния вместо формулы (1) получим

$$
\varepsilon_{n}^{O R G}=\left[1-\exp \left(-\Sigma_{H} h\right)\right]
$$

где $\Sigma_{H}$ - макроскопическое сечение упругого рассеяния нейтронов на ядрах водорода.

Для сцинтилляционных кристаллов KDP следует учесть описанные ранее особенности механизма люминесценции и сцинтилляционного отклика на быстрые нейтроны и сохранить в формуле (1) вклады в сечение $\Sigma_{e l}$ упругого рассеяния не только для протонов отдачи ${ }^{1} \mathrm{H}$, но и для отдачи на легких ядрах кислорода ${ }^{16} \mathrm{O}$. Тогда получим

$$
\varepsilon_{n}^{K D P}=\frac{\Sigma_{H}+\Sigma_{O}}{\Sigma_{H}+\Sigma_{O}+\Sigma_{P}+\Sigma_{K}}\left[1-\exp \left(-\left(\Sigma_{H}+\Sigma_{O}\right) h\right)\right],
$$

где $\Sigma_{H}$ и $\Sigma_{O}$ - сечения упругого рассеяния нейтронов на ядрах водорода и кислорода в составе кристалла KDP, а $\Sigma_{P}$ и $\Sigma_{K}$ - сечения упругого рассеяния на средних ядрах фосфора и калия, отдача которых не вносит существенный вклад в сцинтилляционный сигнал. В формуле (3) также учтено, что вклад неупругих реакций нейтронов в области энергий до $10 \mathrm{MeV}$ в образование вторичных заряженных частиц и импульс сцинтилляций крайне мал.

Для теоретических расчетов использовались данные для усредненных микроскопических сечений взаимодействия нейтронов с ядрами [23]. Спектр источника нейтронов принимался равным спектру источника ${ }^{239} \mathrm{Pu}-\mathrm{Be}[23]$. Концентрация ядер вычисляется по формуле $n_{i}=\rho N_{A}\left(a_{i} / M\right)$, где $N_{A}=6,022 \times 10^{23}$ моль $^{-1}-$ число Авогадро, $\rho$ - плотность вещества, $M$ - молярный вес, $a_{i}$ - число атомов $i$-го сорта в молекуле вещества. Средняя длина свободного пробега нейтронов равна $l_{n}=\Sigma_{t o t}^{-1}$.

В таблице 1 приведены теоретические значения эффективности детектирования быстрых нейтронов, вычисленные по формулам (2)-(3) для типичного пластмассового сцинтиллятора на основе полистирола, органического кристалла антрацена и для кристаллов KDP:Tl и KDP:Ce.

Таблица 1.

Теоретическое сравнение параметров и эффективности детектирования быстрых нейтронов водородсодержащими органическими и неорганическими сцинтилляторами. Толщина сцинтиллятора везде

\begin{tabular}{|c|c|c|c|c|c|}
\hline Сцинтиллятор & $\begin{array}{c}\text { Эффективный } \\
\text { атомный } \\
\text { номер }\end{array}$ & 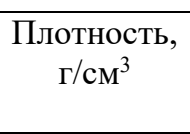 & $\begin{array}{c}\text { Концентрация } \\
\text { ядер H, } \\
\text { см }^{-3}\end{array}$ & $\begin{array}{c}\text { Длина } l_{n}, \\
\text { см }\end{array}$ & $\begin{array}{c}\text { Эффективность } \\
\text { детектирования } \\
\text { (теорет.), \% }\end{array}$ \\
\hline Пластик (полистирол) & 5,7 & 1,04 & $4,82 \times 10^{22}$ & 3,30 & 17,3 \\
\hline Антрацен & 5,8 & 1,25 & $4,23 \times 10^{22}$ & 3,26 & 14,3 \\
\hline KDP сцинтиллятор & 14,3 & 2,34 & $2,07 \times 10^{22}$ & 3,93 & 17,2 \\
\hline
\end{tabular}
принята равной 10 мм.

Проведенные оценки (Таблица 1) указывают на то, что KDP сцинтилляторы могут иметь эффективность регистрации быстрых нейтронов сравнимую с эффективностью детектирования традиционных органических сцинтилляторов.

\section{ЭКСПЕРИМЕНТАЛЬНОЕ ОПРЕДЕЛЕНИЕ ЭФФЕКТИВНОСТИ ДЕТЕКТИРОВАНИЯ БЫСТРЫХ НЕЙТРОНОВ КDР-СЦИНТИЛЛЯТОРАМИ}

Было проведено измерение эффективности регистрации быстрых нейтронов и гамма-квантов кристаллами KDP:Tl и KDP:Се в сравнении со стандартным пластмассовым сцинтиллятором. Для измерений использовались обработанные кристаллы KDP:Tl и KDP:Се размером 10х10х10 мм³ с полированными боковыми гранями. Концентрация активатора соответствовала максимальному световыходу люминесценции. В качестве пластмассового сцинтиллятора использовался сцинтиллятор серии UPS (Институт сцинтилляционных материалов, Харьков, Украина) следующего состава: полистирол 98.5\% (химическая формула $\left.\left(\mathrm{C}_{8} \mathrm{H}_{8}\right)_{\mathrm{n}}\right)+$ люминофор РТР 1,48\% (р-терфенил) + спектросмещающая добавка РОРОР 0,02\%. Исследуемый сцинтиллятор размещался в светозащитном корпусе диаметром 20 мм с диффузной отражающей пленкой типа Теtratek. Измерения амплитудных спектров проводились сборкой сцинтиллятор-ФЭУ. В качестве фотоприемника 
использовался ФЭУ Нamamatsu R7056 (спектр фоточувствительности от 185 до 650 нм, максимум при 420 нм). Напряжение на ФЭУ выбиралось равным 760 В. Импульсы регистрировались с помощью многоканального анализатора. Нижний порог отсечения импульсов 25-30 кэВ. В состав спектрометра входил быстрый предусилитель. Постоянная времени интегрирования сигнала выбиралась для всех сцинтилляторов равной $\sim 6$ мкс.

$\mathrm{B}$ качестве источника нейтронов использовался ${ }^{239} \mathrm{Pu}-\mathrm{Be}$ источник (энергии нейтронов от 0,1 до 10 МэВ, средняя энергия нейтронов $E_{n}=4,2$ МэВ). Расстояние между центрами источника нейтронов и детектора составило $20 \mathrm{~cm}$. Для защиты от сопутствующего гамма-излучения (фотоны с $E_{\gamma} \sim 59$ кэВ от примеси ${ }^{241} \mathrm{Am}$, возникающей из продуктов распада ${ }^{239} \mathrm{Pu}$, а также высокоэнергетические кванты с $E_{\gamma} \sim 0,1-4$ МэВ из других реакций) между источником нейтронов и детектором размещался свинцовый экран толщиной 50 мм (пропускание потока быстрых нейтронов было 45\%). Для защиты от тепловых нейтронов использовался экран из кадмиевой фольги толщиной 1 мм. Структурная схема эксперимента приведена на рис. 2.

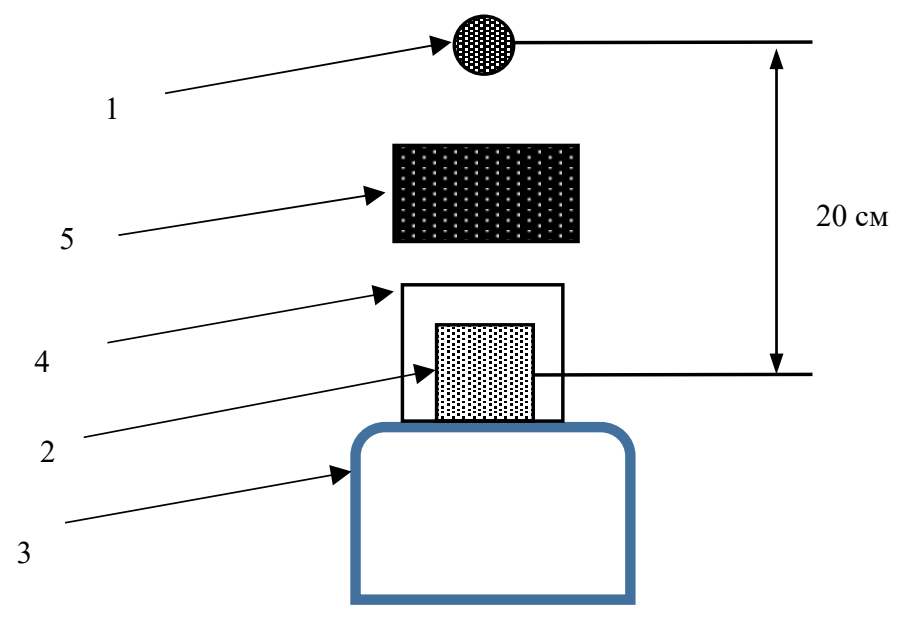

Рис. 2. Схема эксперимента

1 - ${ }^{239} \mathrm{Pu}-$ Ве источник нейтронов; 2 - сцинтиллятор в светозащитном корпусе; 3 - ФЭУ; 4 - Сd-экран от тепловых нейтронов; 5 - Рb-экран от гамма излучения.

На рис. 3 приведены аппаратные спектры исследованных образцов сцинтилляторов при облучении источником нейтронов.

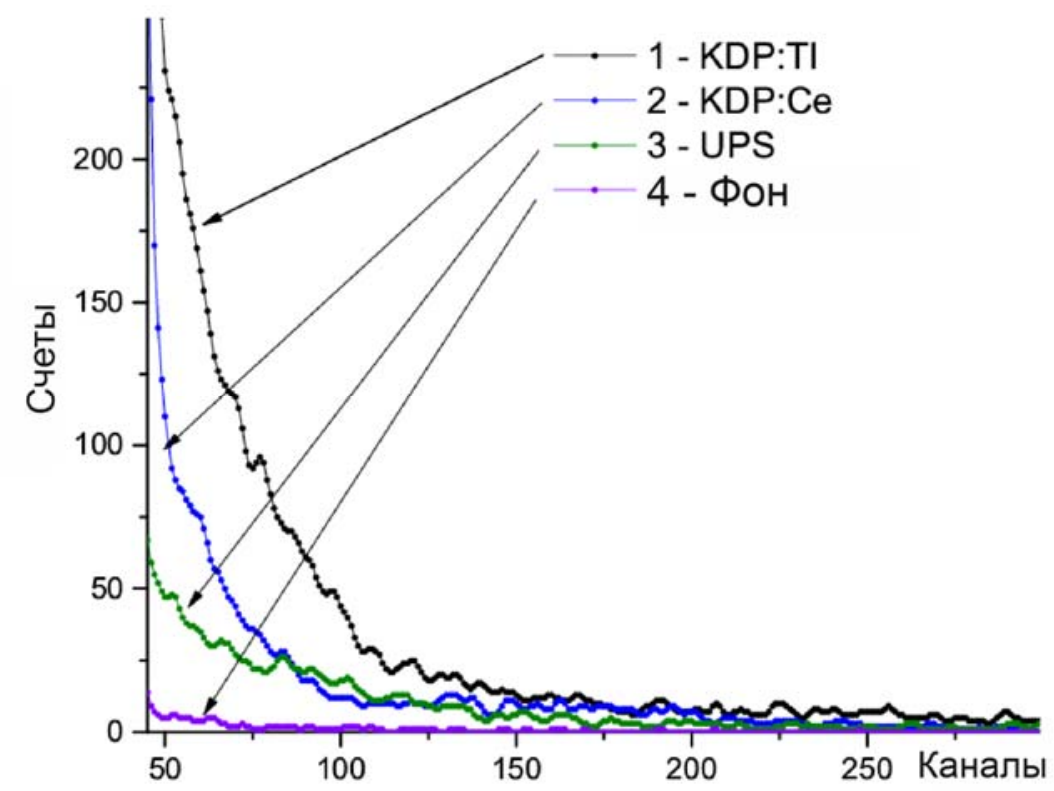

Рис. 3. Амплитудные спектры от ${ }^{239} \mathrm{Pu}-$ Ве источника быстрых нейтронов, полученные для сцинтилляторов 1 - KDP:T1+; 2 - KDP:Cе ${ }^{3+} ; 3$ - полистирол UPS; 4 - естественный фон. Естественный фон из измеренных спектров отклика не вычитался.

На рис. 4 приведены аппаратные спектры при облучении этих же образцов источником гамма-квантов 
${ }^{137} \mathrm{Cs}$. Калибровка энергетической шкалы спектрометра выполнялась с применением этого источника. Максимум отклика $E_{\gamma}=662$ кэВ соответствует 3000 каналу спектрометра. Канал 300 соответствует $E_{\gamma}=60$ кэВ.

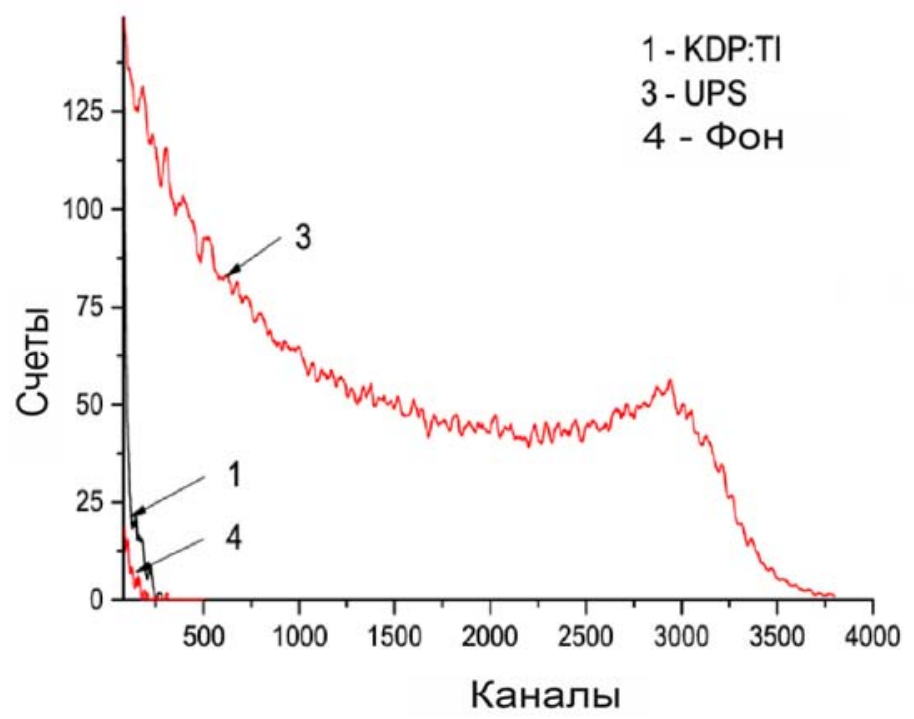

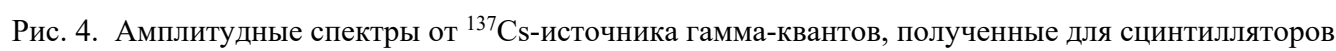
1 - KDP:Tl+; 2 - KDP:Ce $\mathrm{C}^{3+} ; 3$ - полистирол UPS; 4 - естественный фон для KDP. Естественный фон из измеренных спектров отклика не вычитался.

Из рис. 3 следует, что аппаратный отклик на нейтроны для сцинтилляторов KDP:Tl и KDP:Ce распространяется вплоть до энергий 300 кэВ и соответствует непрерывному спектру протонов отдачи. Эти нейтронные спектры подобны спектру для сцинтиллятора UPS на основе полистирола. Отклик на гаммакванты для KDP:Tl, как видно из рис. 4, наоборот, сосредоточен только в низкоэнергетичной области (менее 50 кэВ) и сильно обрезается в области высоких энергий в отличие от спектра пластикового сцинтиллятора. Кроме того, амплитуда отклика на гамма-кванты для KDP-сцинтилляторов в низкоэнергетической области сравнима с вкладом фонового излучения и примерно на порядок ниже, чем у пластика.

На основе измеренных амплитудных спектров была определена эффективность регистрации быстрых нейтронов и $\gamma$-квантов, представленная в таблице 2. Порог регистрации составил $~ 25-30$ кэВ, 50 канал (рис. 3 ).

Таблица 2.

Эффективность регистрации быстрых нейтронов и $\gamma$-квантов сцинтилляторами KDP:T1 ${ }^{+}$и KDP:Ce ${ }^{3+}$ размером

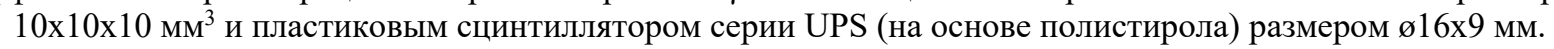

\begin{tabular}{|l|c|c|c|}
\hline \multicolumn{1}{|c|}{ Scintillator } & $\begin{array}{c}\text { Fast neutrons detection } \\
\text { efficiency, } \varepsilon_{n}\end{array}$ & $\begin{array}{c}\text { Gamma detection } \\
\text { efficiency, } \varepsilon_{\gamma} \%\end{array}$ & Relation, $\varepsilon_{n} / \varepsilon_{\gamma}$ \\
\hline Plastic (polystyrene) & $30,0 \%$ & $7,1 \%$ & 4,2 \\
\hline $\mathrm{KDP}: \mathrm{Tl}^{+}(0,1$ wt. $\% \mathrm{Tl})$ & $12,4 \%$ & $0,4 \%$ & 31,0 \\
\hline $\mathrm{KDP}: \mathrm{Ce}^{3+}(0,01$ wt. $\% \mathrm{Ce})$ & $16,2 \%$ & $0,6 \%$ & 27,0 \\
\hline
\end{tabular}

Как видно из таблицы 2, эффективность регистрации быстрых нейтронов сцинтилляторами $\mathrm{KDP}_{\text {: }} \mathrm{Tl}^{+}$ и KDP: $\mathrm{Ce}^{3+}$ варьируется от 12 до $16 \%$ и находится в согласии с теоретическим расчетом (таблица 1). Вместе с тем $n / \gamma$ отношение эффективностей регистрации быстрых нейтронов и гамма-частиц для KDP:T1 и KDP:Cе сцинтилляторов примерно в 7 раз выше, чем у пластика. Это подтверждает высокую естественную селективную чувствительность KDP сцинтилляторов к быстрым нейтронам. При подходящем выборе низкоэнергетического порога для спектрометра регистрация этими сцинтилляторами быстрых нейтронов в смешанных радиационных полях практически не требует применения пассивной защиты от высокоэнергетических $\gamma$-квантов. В детекторах на основе кристаллов KDP:Tl и KDP:Се можно достигнуть $n / \gamma$ дискриминации вплоть до $10^{2}$, что сравнимо с применением активной защиты (по схеме антисовпадений) от комптоновских $\gamma$-квантов. Возможно, этого будет недостаточно для обеспечения современных стандартов [24] обнаружения делящихся и радиоактивных материалов, где требуется разделение сигналов на уровне $10^{5}-10^{6}$. Однако сам факт такого естественного $n / \gamma$ разделения для детектора быстрых нейтронов на основе активированных кристаллов КDP является уникальным и может быть востребован в различных приложениях. 


\section{ВЫВОДЫ}

В работе были исследованы сцинтилляционные отклики кристаллов KDP:Tl и KDP:Се при облучении источниками быстрых нейтронов и гамма-излучения. Обнаруженная люминесценция может быть вызвана прямым возбуждением ионизирующим излучением ионов активаторов, а также (в гораздо большей степени) рекомбинационным механизмом передачи электронных возбуждений кристаллической решетки на центры свечения с участием радиационных дефектов подвижной (при комнатных температурах) водородной подрешетки. Получена теоретическая и измерена экспериментальная эффективность детектирования быстрых нейтронов на основе механизма упругого рассеяния и отдачи на легких ядрах. Эффективность регистрации быстрых нейтронов сцинтилляционными кристаллами KDP толщиной 10 мм варьируется в пределах $12-16 \%$, что сравнимо с эффективностью регистрации для органических сцинтилляторов. Обнаружена высокая естественная селективная чувствительность кристаллов KDP при регистрации быстрых нейтронов по отношению к сопутствующему гамма-излучению. Отношение эффективностей при внутреннем $n / \gamma$ разделении сигналов достигает величины 230 , тогда как для других неорганических сцинтилляторов оно обычно не превосходит 2-3. Таким образом, гамма-излучение регистрируется детекторами на основе KDPсцинтилляторов на уровне естественного фона.

Полученные результаты показывают, что новые селективно-чувствительные к быстрым нейтронам активированные кристаллы KDP:Tl и $\mathrm{KDP}: \mathrm{Ce}$ существенно дополняют перечень твердотельных сцинтилляторов для детектирования нейтронов и могут быть востребованы в различных приложениях, в том числе для контроля делящихся ядерных материалов и антитеррористической безопасности.

\section{СПИСОК ЛИТЕРАТУРЫ}

1. Marion J.B., Fowler J.L. Fast Neutron Physics. Part 1: Techniques, Part 2: Experiments and Theory. - New York, 1960.

2. Harvey J.A., Hill N.W. Scintillation detectors for fast neutron physics // Nuclear Instruments and Methods. - 1979. - Vol. 162. P. 507-530.

3. Klein H., Brooks F.D. Scintillation detectors for fast neutrons // Proc. of Sciences, Int. Workshop on Fast Neutron Detectors and Applications (FNDA 2006), University of Cape Town, South Africa, (April 3-6, 2006), Vol. PoS(FNDA2006)097. - P. 1-24.

4. Kouzes R.T., Lintereur A.T., Siciliano E.R. Progress in alternative neutron detection to address the helium-3 shortage // Nuclear Instruments and Methods in Physics Research, Section A. - 2015. - Vol. A784. - P. 172-175.

5. Ryzhikov V.D., Naydenov S.V., Piven L.A., Onyshchenko G.M., Smith C.F., Pochet T. Fast neutron detectors and portal monitors based on solid-state heavy-oxide scintillators // Radiation Measurements. - 2017. -Vol. 105. - P. 17-25.

6. Beckurts K-H., Wirtz K. Neutron Physics. - Berlin: Springer-Verlag, 1964.

7. Birks J.B. The theory and practice of scintillation counting. - London: Pergamon Press, 1964.

8. Knoll G.F. Radiation detection and measurement, $-4^{\text {th }}$ Ed. - Wiley, 2010.

9. Hull G., Zaitseva N., Cherepy N., Newby J. et al. New organic crystals for pulse shape discrimination // IEEE Trans. Nucl. Sci. - 2009. - Vol. 56. - P. 899-903.

10. Favalli A., Iliev M., Chung K., Hurlbut C. et al. Pulse shape discrimination properties of neutron-sensitive organic scintillators // IEEE Trans. Nucl. Sci. - 2013. - Vol. 60. - P. 1053-1056.

11. Voronov A., Salo V., Puzikov V., Tkachenko V., Vydai Yu. Potassium and ammonium dihydrogen phosphates activated with thallium: growth and luminescence and scintillation properties // Crystallography Reports. - 2006. - Vol. 51. - P. 696-701.

12. Voronov A.P., Vyday Y.T., Salo V.I., Puzikov V.M., Bondarenko S.I. Influence of thallium doping on scintillation characteristics of mixed KDP/ADP crystals // Radiation Measurements. - 2007. - Vol. 42 (4). - P. 553-556.

13. Voronov A.P., Babenko G.N., Puzikov V.M., Roshal A.D., Salo V.I. Doping of KDP single crystals with Cerium: growth and optical properties // Crystallography Reports. - 2008. - Vol. 53. - No. 4. - P. 708-712.

14. Voronov A.P., Babenko G.N., Glushkova L.V., Puzikov V.M., Roshal A.D., Salo V.I. Potassium dihydrogen phosphate doped organic complexes of rare earth elements // Inorganic Materials. - 2009. - Vol. 45. - P. 533-537.

15. Zaitseva N., Carman L. Rapid growth of KDP-type crystals. Progress in crystal growth and characterization of materials. Pergammon, 2001. - P. 1-118.

16. Kolybayeva M., Pritula I., Salo V., Garnov S. Effect of radiation on the properties of water soluble crystals // Proc. SPIE 3244 , Laser-Induced Damage in Optical Materials, 1997 (20 April 1998). - P. 130.

17. Sugimotoy H., Ikeda S.J. Proton transfer in hydrogen-bonded crystalline KH2PO4 // J. Phys.: Condens. Matter. - 1996. - Vol. 8. - P. 603-618.

18. Zazubovich S., Voloshinovskii A., Stryganyuk G. Luminescence of CsCl: Tl crystal under synchrotron excitation // Physica Status Solidi. - 2002. - Vol. 233. - No. 2. - P. 238.

19. Fujita I. Optical-absorption studies on noncubic $\mathrm{s}^{2}$ centers in single crystals of KH2PO4 and RbH2PO4 // Physical Review B. 1994. - Vol. 49. - P. 6462-6469.

20. Ogorodnikov I., Pustovarov V. Electronic excitation energy transfer and nonstationary processes in KH2PO4:Tl crystals // J. Exp. Theor. Phys. - 2017. - Vol. 124. - P. 592-603.

21. Ogorodnikov I., Kirm M., Pustovarov V., Cheremnykh V. Low-temperature time-resolved vacuum ultraviolet spectroscopy of self-trapped excitons in KH2PO4 crystals // Optics and Spectroscopy. - 2003. - Vol. 95. - P. 385-389.

22. Ogorodnikov I., Kirm M., Pustovarov V. Luminescence of the hydrogen bonded crystals // Radiation Measurements. - 2007. Vol. 42. - P. 746-750.

23. The JENDL (Japanese Evaluated Nuclear Data Library), Japan Atomic Energy Agency (JAEA); http://wwwndc.jaea.go.jp/jendl/j40/j40.html.

24. Kouzes R.T., Ely J.R., Lintereur A.T., Stephens D.L. Neutron detector gamma insensitivity criteria, Technical report PNNL18903. - Pacific Northwest National Laboratory, Richland, WA, USA, 2009. 